Editorial

\title{
Simulation on Extracorporeal Membrane Oxygenation
}

\author{
Poonam Malhotra Kapoor ${ }^{1}$ \\ ${ }^{1}$ Department of Cardiac Anaesthesia, All India Institute of Medical \\ Sciences, New Delhi, India \\ J Card Crit Care 2020;3:55-58
}

"Tell me and I forget, show me and I remember, involve me and I learn"

- Franklin

With advancement in medical technology, the use of extracorporeal membrane oxygenation (ECMO) for patients with refractory respiratory and cardiac failure has experienced resurgence in the last decade. In general, universally ECMO indications, and particularly the venous arterial (VA) ECMO patients, are increasing. ECMO delivery at the appropriate time remains a high-risk clinical activity as it has a complex circuitry, low volume base, and use of large cannulae. The prolonged duration of ECMO in most cases ensues hemorrhagic complications and is not without adverse outcomes.

\section{Advantages of Simulation Programs}

In critical care, when all else fails, ECMO allows a novel approach to the intensive care unit (ICU) team, to manage and combat respiratory failure. ECMO centers and ECMO volume have increased globally multifold over the past decade. Managing critically ill patients is highly challenging due to the complexities involved. All ECMO team members require a comprehensive knowledge of cardiopulmonary physiology, expertise with multiple circuit components, and skills to rapidly respond to potential life-threatening situations. ${ }^{1}$

\section{Simulation-Based ECMO Learning Is Experiential}

According to Kolb's theory of learning, "learning is an experiential process with 'abstract concreteness and active experimentation."'2

This can aid in conceptualization of the event, with effective learning, which is thus experiential. If a stringent medical environment can be created, then after experimental teaching of a scenario, there is a reflection of the experience, which, with active help from a facilitator, can lead

to conceptualization of the event with effective learning ( - Fig. 1A-C).

\section{Interprofessional Education as per the World Health Organization}

The World Health Organization (WHO) defines interprofessional education (IPE) as "when students from two or more professions learn about, from, and with each other to enable effective collaboration and improve health outcomes." ${ }^{3}$ The principles of IPE and crisis resource management (CRM) are of paramount importance in ECMO emergencies.

Maintaining values and ethics, with mutual respect for interprofessional practice, forms the mainstay of IPE. Teaching the art of communication forms the backbone of learning in simulation-based ECMO crisis management training. ACRM, which stands for anticipation/planning, communication, mobilization of resources, and routine reevaluation, are all important for ECMO simulation training. Debriefing requires a safe environment for free communication. ${ }^{4}$

\section{ECMO Education: Current Status and the Future of Simulation}

There is ongoing research to investigate the impact of simulation training on ECMO outcomes. Studies have shown an improvement in confidence and performance in managing ECMO patients after simulation training. Allan et al reported a shorter cannulation time in pediatric extracorporeal cardiopulmonary resuscitation (ECPR) performed by cardiac surgical trainees. Su et al reported a faster ECPR initiation time, and Whitmore et al reported a shorter time to achieve a full ECPR support simulation scenario after simulation training. ${ }^{5,6}$ Recently, Zakhary et al published the first randomized controlled trial in ECMO simulation comparing simulation training with water drill. ${ }^{7}$ The simulation group had a superior performance gauged by

(C)2020 Official Publication of The Simulation Society (TSS), accredited by International Society of Cardiovascular Ultrasound (ISCU).

\section{License terms}

10.1055/s-0040-1710164

ISSN 2457-0206.
Malhotra Kapoor, MD, DNB, MNAMS, FTEE, FIACTA, FISCU, Department of Cardiac Anaesthesia, All India Institute of Medical Sciences, Ansari Nagar, New Delhi 110029, India (e-mail: drpoonamaiims@gmail.com).

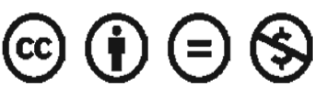



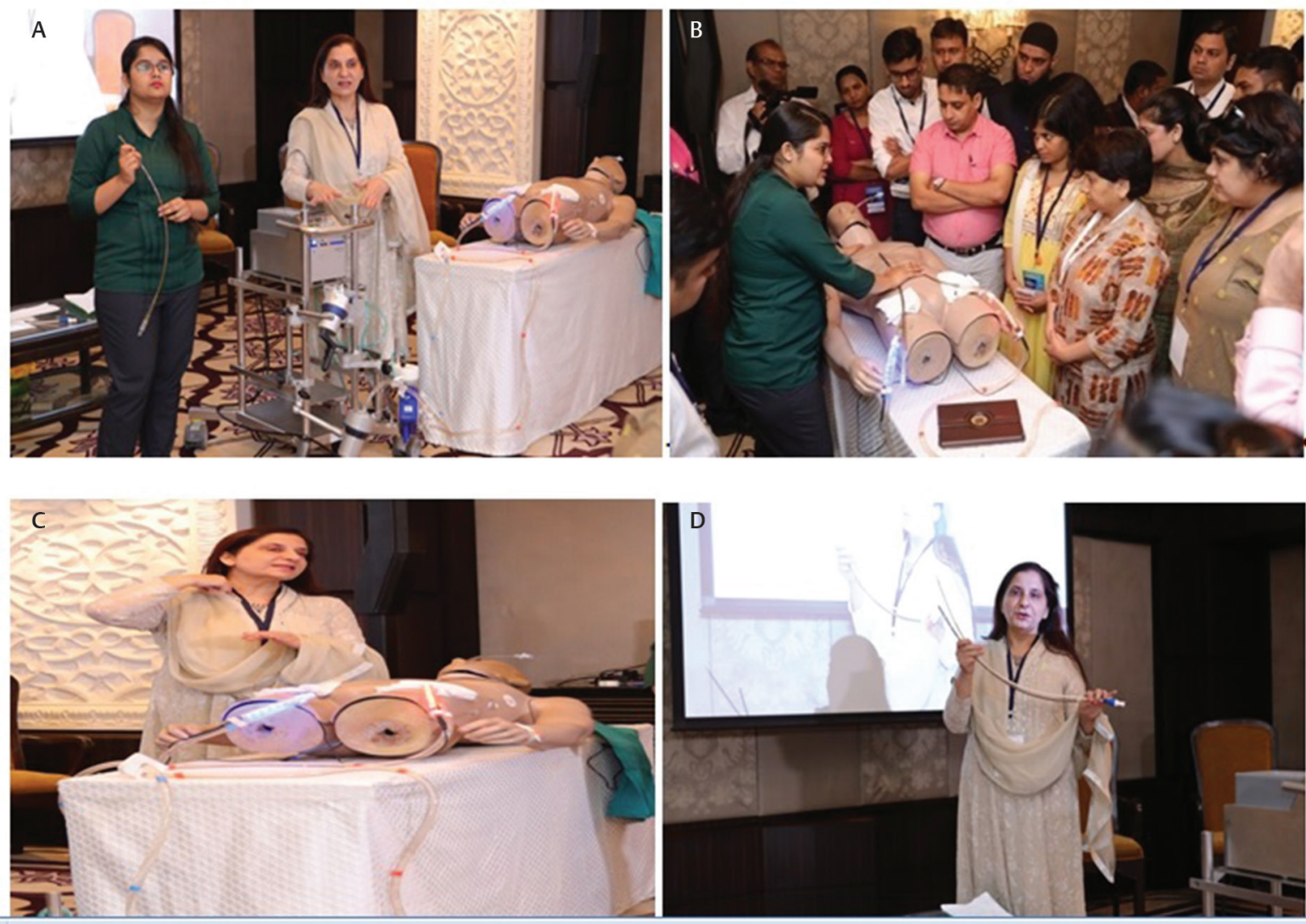

Fig. 1 (A) An extracorporeal membrane oxygenation (ECMO) simulation setup at AllMS with types of cannulae. (B) Site of Insertion. (C) Complication. (D) Debriefing sessions at an ECMO cannulation hands on experience.
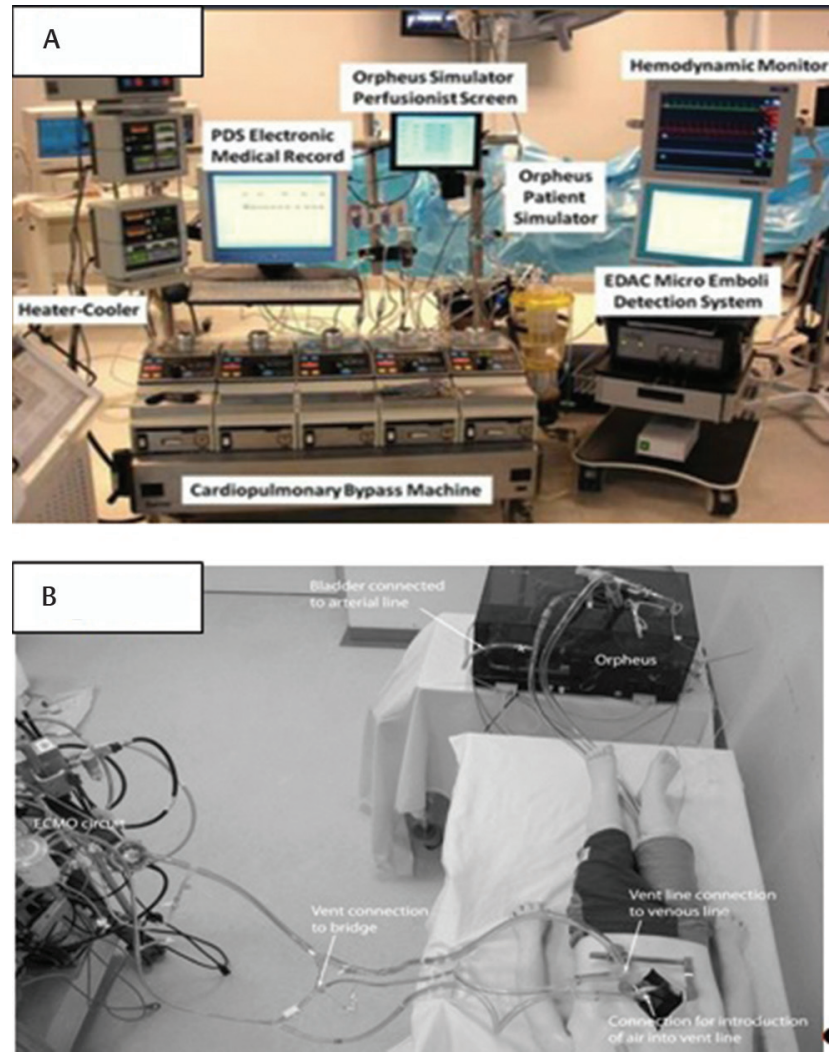

Fig. 2 (A, B) The system is made up of the Orpheus perfusion simulator incorporating a modified resuscitation mannequin between the hydraulic module of the Orpheus and the extracorporeal membrane oxygenation system. achieving a higher scenario score and a shorter time to critical action. Notably, the superior performance was sustained at a 1-year evaluation? (-Figs. 2A, B and 3).

\section{Orpheus Perfusion Simulator}

\section{A modern ECMO Simulation Setup ECMO Simulator Setup}

The setup includes the following: ECMO mannequin, monitors (virtual ventilator, virtual ECMO system, and virtual patient monitor), and iPads (trainer and trainee). All the data in the monitors can be integrated and projected on to a bigger screen to benefit a larger audience. It is vital to use the simulation props to recreate a simulation room imitating the ICU setup as realistically as possible.

\section{Understand the Practical Requirements}

The cannulation task trainer's aim is to make participants understand the practical requirements (cannulation tray, cannula availability, etc.) and developing muscle memory on vessel identification, sizing, Seldinger technique, positioning, de-airing, connection to ECMO circuit (-Fig. 4A-D).

\section{Connection to ECMO Circuit}

\section{Mannequin Design}

The ECMO mannequin is designed to simulate an adult patient on ECMO. It serves as a task trainer to practice. With the Percutaneous technique of cannulation 

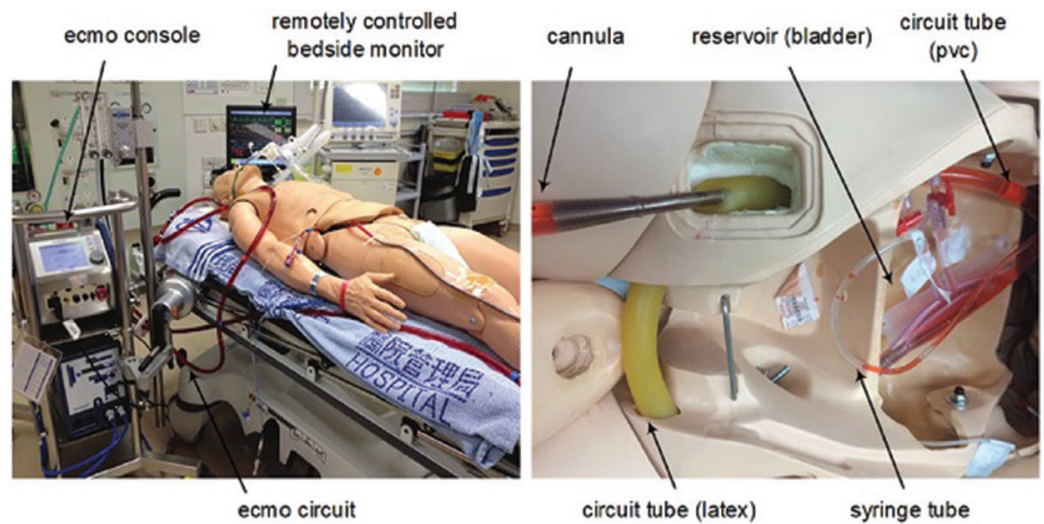

Fig. 3 A modern extracorporeal membrane oxygenation (ECMO) simulation setup. It is currently used with modern centrifugal pump-based ECMO circuits such as the Maquet Rotaflow pump, the Maquet CardioHelp system, and Thoratec CentriMag, in addition to a plethora of basic to advanced mannequins such as the Laerdal ResuciJunior, SimMan, SimBaby, Nursing Kelly, CAE PediaSim, and Gaumard Hal.
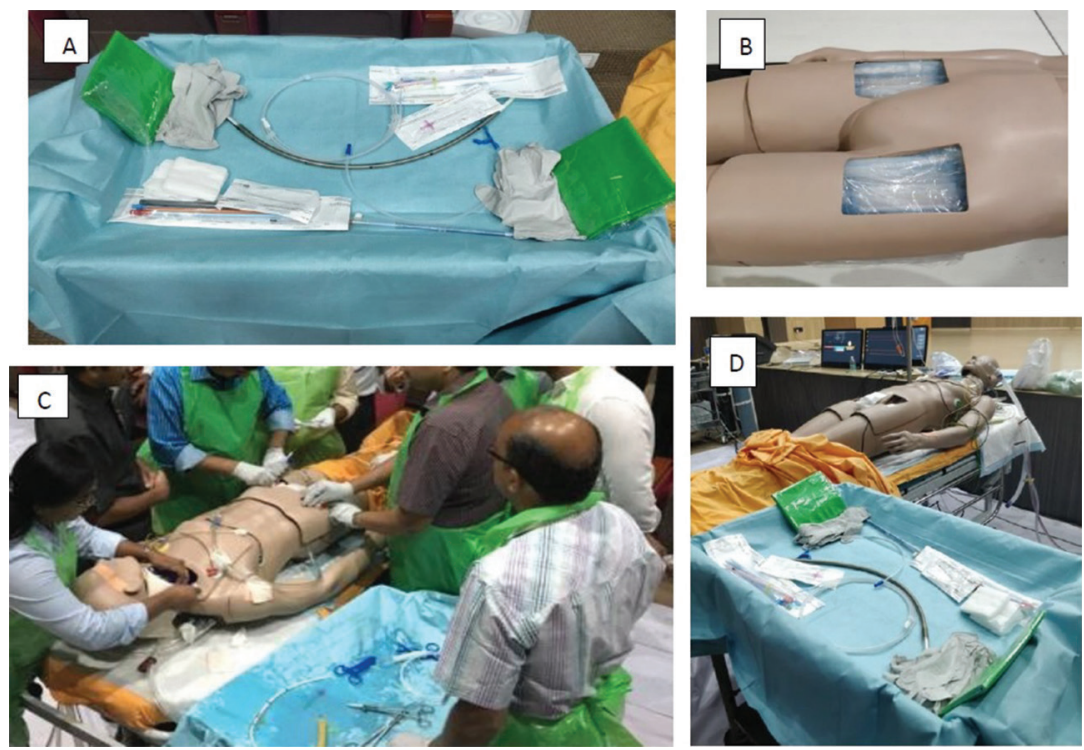

Fig. 4 (A) Cannulation tray set up for task trainer. (B) Cannulation access on the mannequin. (C) Cannulation in practice. (D) The full setup.

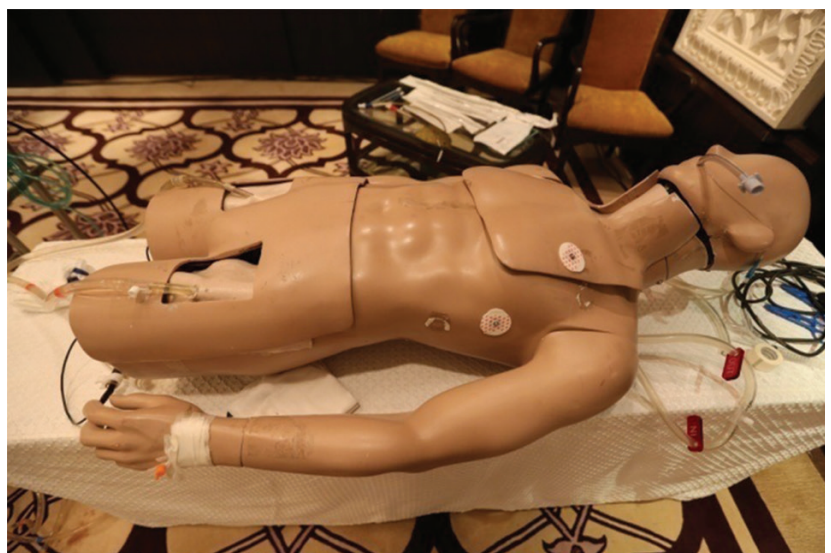

Fig. 5 Maquet simulator used by The Simulation Society.

for femoral and jugular approaches, it is also possible to enable ultrasound-guided cannulation for vessel sizing and safe puncture. The femoral and carotid pulses are palpable, and intensity can be varied to simulate various clinical situations ( $\mathbf{- F i g s .} \mathbf{5}$ and $\mathbf{6}$ ).

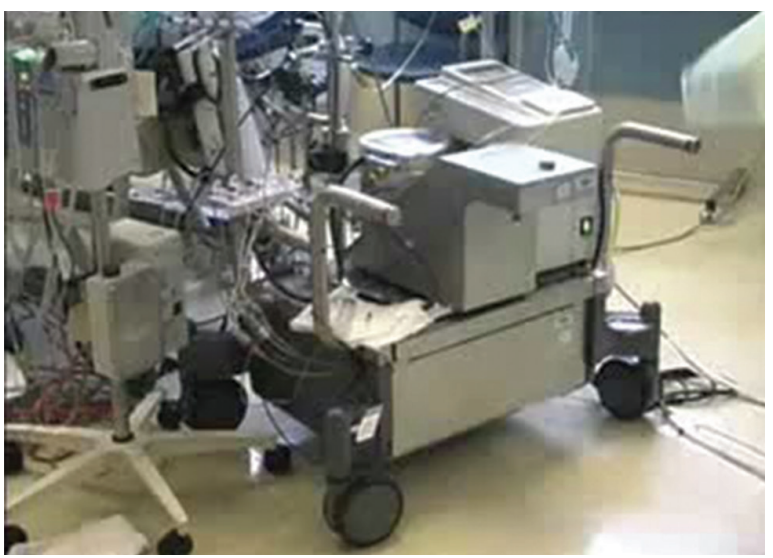

Fig. 6 Extracorporeal membrane oxygenation setup in the operating room.

\section{ECMO Simulation Mannequin}

The mannequin has inbuilt ECMO circuits for simulation (-Fig. 5). Both venovenous and VA scenarios along with distal perfusion can be simulated. The setup includes - Table 1 . 
Table 1 Essential requirements for ECMO simulation training

\begin{tabular}{|l|l|}
\hline $\begin{array}{l}\text { ECMO mannequin (with inbuilt } \\
\text { simulation circuits, cannulas, and } \\
\text { external reservoir) }\end{array}$ & Vitals virtual monitor \\
\hline Cardiohelp Full System & ECMO virtual monitor \\
\hline HLS kit & SimCast (for projection) \\
\hline Air and oxygen source & Trainer iPad \\
\hline Ventilator & Trainee iPad \\
\hline ICU environment props & \\
\hline
\end{tabular}

\section{Conclusion}

The importance of simulation training in ECMO education curriculum is increasing. Surveys conducted in the United States reported that $46 \%$ of the ECMO centers have an ECMO simulation program and that $73 \%$ of the centers have adopted simulation as part of the institutional ECMO credentialing program. Simulation-based training with the goal of addressing IPE and CRM is especially suited to improve team performance, prevent error, and enhance patient safety. In the aviation industry, where running a randomized controlled trial is impossible, simulation training is very likely the most efficient and appropriate training tool for crisis management in the provision of ECMO care.

\section{Extracorporeal Life Support Organization's Education Program Paves the Way}

The Simulation Society in 2018 was among the first few societies to start an International Online ECMO education fellowship course with simulation-based training. More than 100 students cleared the accredited examination in 2019. The Extracorporeal Life Support Organization in 2019 declared a Global ECMO Education (ECMOED) Taskforce with an international and multidisciplinary group of health care practitioners and educators, who are experts in ECMO teaching and training with international collaborators, there will now with the help of ECMOed Taskforce a standardization of ECMO education, which is today the need of the hour. Implementation of ECMO-accredited courses and workshops are on the horizon for high-quality simulation-based ECMO training worldwide.

The Journal of Cardiac Critical Care (JCCC ${ }^{\text {TSS }}$ )-based second batch of online ECMO TSS course continues to run in 2020 with an exit examination in September 2020, with Indian ECMO experts.

\section{Conflict of Interest}

None.

\section{References}

1 Abrams D, Garan AR, Abdelbary A, et al; International ECMO Network (ECMONet) and The Extracorporeal Life Support Organization (ELSO). Position paper for the organization of ECMO programs for cardiac failure in adults. Intensive Care Med 2018;44(6):717-729

2 Kolb DA. Experiential Learning: Experience as the Source of Learning and Development. Upper Saddle River, NJ: Prentice Hall; 1984

3 World Health Organization. Framework for Action on Interprofessional Education \& Collaborative Practice. Available at: https:// apps.who.int/iris/bitstream/handle/10665/70185/WHO_HRH_ HPN_10.3_eng.pdf;jses. Accessed December 30, 2019

4 Gardner R. Introduction to debriefing. Semin Perinatol 2013;37(3):166-174

5 Allan CK, Pigula F, Bacha EA, et al. An extracorporeal membrane oxygenation cannulation curriculum featuring a novel integrated skills trainer leads to improved performance among pediatric cardiac surgery trainees. Simul Healthc 2013;8:221-228

6 Su L, Spaeder MC, Jones MB, et al. Implementation of an extracorporeal cardiopulmonary resuscitation simulation program reduces extracorporeal cardiopulmonary resuscitation times in real patients. Pediatr Crit Care Med 2014;15:856-860

7 Zakhary BM, Kam LM, Kaufman BS, Felner KJ. The utility of high-fidelity simulation for training critical care fellows in the management of extracorporeal membrane oxygenation emergencies: a randomized controlled trial. Crit Care Med 2017;45(8):1367-1373 